J. Korean Math. Soc. 50 (2013), No. 4, pp. 715-725

http://dx.doi.org/10.4134/JKMS.2013.50.4.715

\title{
NUMERICAL METHOD FOR A 2NTH-ORDER BOUNDARY VALUE PROBLEM
}

\author{
Chenmei Xu, Shuai Jian, and Bo Wang
}

\begin{abstract}
In this paper, a finite difference scheme for a two-point boundary value problem of 2 nth-order ordinary differential equations is presented. The convergence and uniqueness of the solution for the scheme are proved by means of theories on matrix eigenvalues and norm. Numerical examples show that our method is very simple and effective, and that this method can be used effectively for other types of boundary value problems.
\end{abstract}

\section{Introduction}

Assume that a 2 nth-order $(n \geq 2)$ two-point boundary valve problem (BVP) is given in the form

$$
\begin{cases}y^{(2 n)}=f(x) y+g(x), & 0<x<1 \\ y^{(2 \alpha)}(0)=a_{\alpha}, & \alpha=0,1, \ldots, n-1, \\ y^{(2 \alpha)}(1)=b_{\alpha}, & \end{cases}
$$

where $f(x)$ and $g(x)$ are continuous functions on the closed interval $[0,1]$ and $a_{\alpha}, b_{\alpha}$ are all real constants. The problem (1.1) frequently occurs in structural engineering, astrophysics and other branches of physical sciences. The problem (1.1) has a unique analytic solution for a fixed positive integer $n \geq 2$ under the general condition [5]

$$
f(x) \neq(-1)^{n} j^{2 n} \pi^{2 n}, j=1,2, \ldots, x \in[0,1] .
$$

In general, the analytic solution of the system (1.1) under the condition (1.2) cannot be obtained, and the numerical methods are required. Some finite difference methods [2,6,9] of orders $O\left(h^{2}\right), O\left(h^{4}\right)$ and $O\left(h^{6}\right)$ were derived under the condition $-36 \leq f(x) \leq 0, x \in[0,1]$ for $n=2$. Then some $O\left(h^{2}\right)$

Received April 12, 2010; Revised February 19, 2012.

2010 Mathematics Subject Classification. 65L10, 65L70.

Key words and phrases. boundary valve problem, finite difference scheme, 2nth-order ordinary differential equation, total truncation error.

The work was supported by the Natural Science Foundation of the Education Department of Henan Province(2010A1000030) and National Natural Science Foundation of China(61201253). 
and $O\left(h^{\frac{3}{2}}\right)$ methods $[7,8,10]$ were obtained for $n=2$ under the hypotheses $f(x) \leq 0, x \in[0,1]$ and $|f(x)| \leq \pi^{4}, x \in[0,1]$, respectively. Later, an $O\left(h^{\frac{3}{2}}\right)$ method [11] for $n=2$ was obtained under the condition $f(x) \neq j^{4} \pi^{4}, j=$ $1,2, \ldots, x \in[0,1]$. Other methods $[1,3,4,12,13]$ were introduced recently. However, the numerical methods for the problem (1.1) under the condition (1.2) has not yet been studied.

The main purpose of this paper is to set up a finite difference scheme for the $\operatorname{BVP}(1.1)(n \geq 2)$ and discuss its convergence and uniqueness of the solution for the scheme under the condition (1.2). Also, a more general domain $[a, b]$ can be transformed into the standard domain $[0,1]$ through a straightforward substitution $x=\frac{t-a}{b-a}$. Thus the problem and condition discussed in this paper are quite general. This scheme is very simple to implement and efficient. Besides, the method can be applied to the study of some nonlinear problems, which arise frequently in many areas of engineering.

This paper is organized as follows. In Section 2, we introduce a finite difference scheme for the BVP (1.1). In Section 3, we analyze the convergence and uniqueness of the solution for the scheme under the condition (1.2) by using theories on eigenvalues and norm. In Section 4, the convergence of our method is illustrated by some numerical examples.

\section{Finite difference scheme}

Let $N$ be a positive integer and $N \geq 2 n$. Divide the interval $[0,1]$ into $N+1$ parts. Then the mesh step size is $h=\frac{1}{N+1}$ and the mesh points are $x_{i}=i h$ for $i=0,1, \ldots, N+1$. For simplicity, we denote that $y_{i}=y\left(x_{i}\right), f_{i}=f\left(x_{i}\right), g_{i}=$ $g\left(x_{i}\right)$.

By means of the Taylor's formula, we obtain

$$
\left\{\begin{aligned}
-2 y_{1}+y_{2} & =h^{2} y_{1}^{\prime \prime}-y_{0}+t_{1}, \\
y_{i-1}-2 y_{i}+y_{i+1} & =h^{2} y_{i}^{\prime \prime}+t_{i}, i=2,3, \ldots, N-1, \\
y_{N-1}-2 y_{N} & =h^{2} y_{N}^{\prime \prime}-y_{N+1}+t_{N},
\end{aligned}\right.
$$

where the truncation errors are

$$
t_{i}=\frac{2}{4 !} h^{4} y^{(4)}\left(\bar{x}_{i}\right), x_{i-1}<\bar{x}_{i}<x_{i+1}, i=1,2, \ldots, N .
$$

By (2.1), the discrete form of the BVP (1.1) for $n=1$ can be expressed as

$$
\left\{\begin{aligned}
-2 y_{1}+y_{2} & =h^{2} f_{1} y_{1}+h^{2} g_{1}-y_{0}+t_{1} \\
y_{i-1}-2 y_{i}+y_{i+1} & =h^{2} f_{i} y_{i}+h^{2} g_{i}+t_{i}, \quad i=2,3, \ldots, N-1, \\
y_{N-1}-2 y_{N} & =h^{2} f_{N} y_{N}+h^{2} g_{N+1}-y_{N+1}+t_{N} .
\end{aligned}\right.
$$

The equation (2.2) can be written in the matrix form

$$
P Y=h^{2} D Y+C_{1}+T_{1}
$$


where

$$
P=\left(\begin{array}{ccccccc}
-2 & 1 & 0 & \cdots & 0 & 0 & 0 \\
1 & -2 & 1 & \cdots & 0 & 0 & 0 \\
\vdots & \vdots & \vdots & & \vdots & \vdots & \vdots \\
0 & 0 & 0 & \cdots & 1 & -2 & 1 \\
0 & 0 & 0 & \cdots & 0 & 1 & -2
\end{array}\right)
$$

is a tri-diagonal matrix of order $N, Y=\left(y_{1}, y_{2}, \ldots, y_{N}\right)^{T}, D=\operatorname{diag}\left(f_{1}, f_{2}\right.$, $\left.\ldots, f_{N}\right), T_{1}=\left(t_{i}\right)=\left(O\left(h^{4}\right)\right)$, and $C_{1}=\left(c_{i}\right)=\left(g_{1} h^{2}-a_{0}, g_{2} h^{2}, \ldots, g_{N-1} h^{2}\right.$, $\left.g_{N} h^{2}-b_{0}\right)^{T}$.

Similarly, based on the Taylor's formula, we have the relationships at $x_{i}$

$$
y_{i-1}^{\prime \prime}-2 y_{i}^{\prime \prime}+y_{i+1}^{\prime \prime}=h^{2} y_{i}^{(4)}+\frac{2}{4 !} h^{4} y^{(6)}\left(\bar{x}_{i 1}\right), \quad x_{i-1}<\bar{x}_{i 1}<x_{i+1}
$$

$$
y_{i-1}^{(4)}-2 y_{i}^{(4)}+y_{i+1}^{(4)}=h^{2} y^{(6)}\left(\bar{x}_{i 2}\right), \quad x_{i-1}<\bar{x}_{i 2}<x_{i+1} .
$$

Suppose that the value of the sixth derivative $y^{(6)}(x)$ on the interval $\left[x_{i-2}\right.$, $x_{i+2}$ ] does not change too rapidly. From (2.4) and (2.5), we can find a value $\bar{x}_{i}$ that lies in $\left[x_{i-2}, x_{i+2}\right]$ so that

$$
\begin{aligned}
& y_{i-2}-4 y_{i-1}+6 y_{i}-4 y_{i+1}+y_{i+2}=h^{4} y_{i}^{(4)}+\frac{1}{6} h^{6} y^{(6)}\left(\bar{x}_{i}\right), \\
& x_{i-2}<\bar{x}_{i}<x_{i+2}, i=2,3, \ldots, N-1 .
\end{aligned}
$$

By (2.6), we have the relationships

$$
\left\{\begin{array}{l}
5 y_{1}-4 y_{2}+y_{3}=2 y_{0}-h^{2} y_{0}^{\prime \prime}+h^{4}\left(-\frac{1}{12} y_{0}^{(4)}+y_{1}^{(4)}\right)+t_{1} \\
y_{i-2}-4 y_{i-1}+6 y_{i}-4 y_{i+1}+y_{i+2}=h^{4} y_{i}^{(4)}+t_{i}, i=2,3, \ldots, N-1 \\
y_{N-2}-4 y_{N-1}+5 y_{N}=2 y_{N+1}-h^{2} y_{N+1}^{\prime \prime}+h^{4}\left(y_{N}^{(4)}-\frac{1}{12} y_{N+1}^{(4)}\right)+t_{N}
\end{array}\right.
$$

where the truncation errors are

$$
\begin{aligned}
t_{1} & =\frac{59}{360} h^{6} y^{(6)}\left(\overline{x_{1}}\right), x_{0}<\overline{x_{1}}<x_{3}, \\
t_{i} & =\frac{1}{6} h^{6} y^{(6)}\left(\overline{x_{i}}\right), x_{i-2}<\overline{x_{i}}<x_{i+2}, i=2,3, \ldots, N-1, \\
t_{N} & =\frac{59}{360} h^{6} y^{(6)}\left(\overline{x_{N}}\right), x_{N-2}<x_{N}<x_{N+1} .
\end{aligned}
$$

From (2.7), we see that the discrete form of the BVP (1.1) for $n=2$ is

$$
\left\{\begin{array}{l}
5 y_{1}-4 y_{2}+y_{3}=2 y_{0}-h^{2} y_{0}^{\prime \prime}+h^{4}\left(-\frac{1}{12} y_{0}^{(4)}+f_{1} y_{1}+g_{1}\right)+t_{1} \\
y_{i-2}-4 y_{i-1}+6 y_{i}-4 y_{i+1}+y_{i+2}=h^{4}\left(f_{i} y_{i}+g_{i}\right)+t_{i}, i=2,3, \ldots, N-1, \\
y_{N-2}-4 y_{N-1}+5 y_{N}=2 y_{N+1}-h^{2} y_{N+1}^{\prime \prime}+h^{4}\left(f_{N} y_{N}+g_{N}-\frac{1}{12} y_{N+1}^{(4)}\right)+t_{N} .
\end{array}\right.
$$


The equation (2.9) can be written in the matrix form

$$
P^{2} Y=h^{4} D Y+C_{2}+T_{2},
$$

where $T_{2}=\left(t_{i}\right)=\left(O\left(h^{6}\right)\right)$ from $(2.8)$, and

$$
C_{2}=\left(c_{i}\right)=\left(\begin{array}{c}
g_{1} h^{4}+2 a_{0}-a_{1} h^{2}-\frac{1}{12} h^{4}\left(f_{0} a_{0}+g_{0}\right) \\
g_{2} h^{4}-a_{0} \\
g_{3} h^{4} \\
\ldots \\
g_{N-2} h^{4} \\
g_{N-1} h^{4}-b_{0} \\
g_{N} h^{4}+2 b_{0}-b_{1} h^{2}-\frac{1}{12} h^{4}\left(f_{N+1} b_{0}+g_{N+1}\right)
\end{array}\right) .
$$

Furthermore, the central discrete version of the BVP (1.1) can be given in a similar way as in (2.3) and (2.10), expressed uniformly by the matrix equation

$$
P^{n} Y=h^{2 n} D Y+C_{n}+T_{n},
$$

where $T_{n}=\left(t_{i}\right)=\left(O\left(h^{2 n+2}\right)\right)$ and $C_{n}=\left(c_{i}\right)$ are too complex to present here.

On neglecting the truncation error $T_{n}$ in (2.11), we arrive at the central difference scheme for the BVP (1.1)

$$
P^{n} Z=h^{2 n} D Z+C_{n},
$$

where $Z=\left(z_{i}\right)$ is the approximate solution of (2.11). The solution of the system (2.12) can be calculated by the $L U$ decomposition method, where $L$ and $U$ are a lower triangular and an upper triangular matrices, respectively, or by the chase method and so on. We shall analyze the convergence and uniqueness of the scheme (2.12) under the condition (1.2) in the next section.

\section{Convergence analysis}

\subsection{Some useful conclusions}

If the matrix $P^{n}-h^{2 n} D$ is invertible, then the total truncation error of the scheme (2.12) is

$$
E=\left(e_{i}\right)=\left(y\left(x_{i}\right)-z_{i}\right)=Y-Z=\left(P^{n}-h^{2 n} D\right)^{-1} T_{n} .
$$

Lemma 3.1 ([13]). The eigenvalues of the tri-diagonal matrix $P$ introduced in Section 2 are $-4 \sin ^{2}\left(\frac{l \pi h}{2}\right)$ for $l=1,2, \ldots, N$.

Lemma 3.2. Let $A$ be a real symmetric matrix of Nth-order. If there exists $a>0$ such that $\|A \eta\| \geq a\|\eta\|$ for any vector $\eta \in R^{N}$, then $A$ is invertible and $\left\|A^{-1} \eta\right\| \leq \frac{1}{a}\|\eta\|$.

Proof. Denote the eigenvalues of $A$ by $\lambda_{1}, \lambda_{2}, \ldots, \lambda_{N}$ and the corresponding eigenvectors by $\xi_{1}, \xi_{2}, \ldots, \xi_{N}$. Then

$$
\left|\lambda_{l}\right|\left\|\xi_{l}\right\|=\left\|\lambda_{l} \xi_{l}\right\|=\left\|A \xi_{l}\right\| \geq a\left\|\xi_{l}\right\|, l=1,2, \ldots, N .
$$


Thus $\left|\lambda_{l}\right| \geq a$, which means that all of the eigenvalues $\lambda_{l}$ are nonzero and $A$ is invertible consequently.

For the eigenvalues $\lambda_{l}^{-1}$ of $A^{-1}$, we have $0<\left|\lambda_{l}^{-1}\right| \leq \frac{1}{a}$. Denoting by $\rho\left(A^{-1}\right)$ the spectral radius of $A^{-1}$, it yields in view of the symmetry of $A^{-1}$ that [14] $\left\|A^{-1}\right\|=\rho\left(A^{-1}\right) \leq \frac{1}{a}$. Hence, for any vector $\eta \in R^{N}$, it holds that

$$
\left\|A^{-1} \eta\right\| \leq\left\|A^{-1}\right\|\|\eta\| \leq \frac{1}{a}\|\eta\| .
$$

\subsection{Convergence analysis}

Now we will discuss convergence of the above finite difference scheme (2.12). For the sake of simplicity, we will only consider the case when $n$ is odd. The even case can be treated analogously. Now the condition (1.2) can be written as

$$
f(x) \neq-j^{2 n} \pi^{2 n}, j=1,2, \ldots, x \in[0,1] .
$$

Based on Lemma 3.1, the eigenvalues of $P^{n}$ are

$$
\lambda_{l}=-4^{n} \sin ^{2 n}\left(\frac{l \pi h}{2}\right), l=1,2, \ldots, N .
$$

Inserting (3.2) into the well-known inequality

$$
t-\frac{t^{3}}{6} \leq \sin t \leq t, t \in\left(0, \frac{\pi}{2}\right)
$$

we establish the estimate for the eigenvalues of $P^{n}$

$$
-l^{2 n} \pi^{2 n} h^{2 n} \leq \lambda_{l} \leq-l^{2 n} \pi^{2 n} h^{2 n}\left(1-\frac{l^{2} \pi^{2} h^{2}}{24}\right)^{2 n}, l=1,2, \ldots, N .
$$

Set $p=\inf _{x \in[0,1]} f(x)$ and $q=\sup _{x \in[0,1]} f(x)$. Then the condition (1.2) can be considered into two cases by the continuity of $f(x)$ on the interval $[0,1]$ : either

$$
p>-\pi^{2 n},
$$

or there exists an integer $k \geq 1$ such that

$$
-(k+1)^{2 n} \pi^{2 n}<p \leq q<-k^{2 n} \pi^{2 n} .
$$

Since $P^{n}$ is a real symmetric matrix, we can choose an orthogonal matrix $T$ of Nth-order such that

$$
T^{\prime} P^{n} T=\operatorname{diag}\left(\lambda_{1}, \lambda_{2}, \ldots, \lambda_{N}\right) .
$$

For any vector $U=\left(u_{j}\right) \in R^{N}$ and $\tilde{U}=\left(\tilde{u}_{j}\right)=T^{\prime} U$, according to the concept of inner product in $R^{N}$ and (3.2), we have

$$
\begin{aligned}
\left(U,\left(P^{n}-h^{2 n} D\right) U\right) & =\tilde{U}^{\prime} \operatorname{diag}\left(\lambda_{1}, \lambda_{2}, \ldots, \lambda_{N}\right) \tilde{U}-U^{\prime} h^{2 n} D U \\
& \leq\left(\lambda_{1}-p h^{2 n}\right)(U, U) .
\end{aligned}
$$

From (3.4), it follows that

$$
\left(U,\left(P^{n}-h^{2 n} D\right) U\right) \leq-h^{2 n}\left[\pi^{2 n}\left(1-\frac{\pi^{2} h^{2}}{24}\right)^{2 n}+p\right](U, U) .
$$


(1) Suppose that $f(x)$ satisfies (3.5). In view of (3.5), when the step $h$ satisfies

$$
h \leq h_{1}=\frac{2 \sqrt{6}}{\pi}\left[1-\left(\frac{1}{2}-\frac{p}{2 \pi^{2 n}}\right)^{\frac{1}{2 n}}\right]^{\frac{1}{2}},
$$

it holds that $\left|\left(U,\left(P^{n}-h^{2 n} D\right) U\right)\right| \geq \frac{h^{2 n}}{2}\left(p+\pi^{2 n}\right)(U, U)$. Then from $\|U\|^{2}=$ $(U, U)$ and Cauchy-Schwartz inequality, we obtain

$$
\|U\|\left\|\left(P^{n}-h^{2 n} D\right) U\right\| \geq \frac{h^{2 n}}{2}\left(p+\pi^{2 n}\right)\|U\|^{2} .
$$

Furthermore, we have the result

$$
\left\|\left(P^{n}-h^{2 n} D\right) U\right\| \geq \frac{h^{2 n}}{2}\left(p+\pi^{2 n}\right)\|U\| .
$$

Obviously, the matrix $P^{n}-h^{2 n} D$ is real symmetric. So by using Lemma 3.2 and (3.8), we obtain that the matrix $P^{n}-h^{2 n} D$ is invertible and

$$
\left\|\left(P^{n}-h^{2 n} D\right)^{-1} U\right\| \leq \frac{2}{h^{2 n}\left(p+\pi^{2 n}\right)}\|U\| .
$$

(2) Suppose that $f(x)$ satisfies (3.6). Without loss of generality, let $N \geq k+1$ and set $m=\frac{1}{2}(p+q)$. Then we derive

$$
\begin{aligned}
\left\|\left(P^{n}-h^{2 n} D\right) U\right\| & \geq\left\|\left(P^{n}-h^{2 n} m I\right) U\right\|-\left\|\left(h^{2 n} m I-h^{2 n} D\right) U\right\| \\
& \geq\|U\| h^{2 n}\left(\min _{1 \leq i \leq N}\left|\frac{\lambda_{i}}{h^{2 n}}-m\right|-\max _{1 \leq i \leq N}\left|m-f_{i}\right|\right) .
\end{aligned}
$$

For the last term in the bracket of (3.10), it clearly holds that

$$
\max _{1 \leq i \leq N}\left|m-f_{i}\right| \leq \frac{q-p}{2} \text {. }
$$

For $\left|\frac{\lambda_{i}}{h^{2 n}}-m\right|$ in the bracket of (3.10), when the step $h$ satisfies

$$
h \leq h_{2}=\frac{2 \sqrt{6}}{(k+1) \pi}\left\{1-\left[\frac{1}{2}-\frac{p}{2(k+1)^{2 n} \pi^{2 n}}\right]^{\frac{1}{2 n}}\right\}^{\frac{1}{2}},
$$

we have

$$
(k+1)^{2 n} \pi^{2 n}\left[1-\frac{(k+1)^{2} \pi^{2} h^{2}}{24}\right]^{2 n}+m \geq \frac{(k+1)^{2 n} \pi^{2 n}+q}{2}>0 .
$$

Furthermore, in view of $(3.2),(3.4),(3.6)$ and (3.13), it is easily checked that

$$
\left\{\begin{array}{l}
m-\frac{\lambda i}{h^{2 n}} \geq m+(k+1)^{2 n} \pi^{2 n}\left[1-\frac{(k+1)^{2} \pi^{2} h^{2}}{24}\right]^{2 n}>0, N \geq i \geq k+1 . \\
-m+\frac{\lambda i}{h^{2 n}} \geq-m-k^{2 n} \pi^{2 n}>0, k \geq i \geq 1
\end{array}\right.
$$

Therefore, from (3.14), the estimate can be given in the form

$$
\min _{1 \leq i \leq N}\left|\frac{\lambda_{i}}{h^{2 n}}-m\right|
$$




$$
\geq \min \left\{-m-k^{2 n} \pi^{2 n}, m+(k+1)^{2 n} \pi^{2 n}\left[1-\frac{(k+1)^{2} \pi^{2} h^{2}}{24}\right]^{2 n}\right\} .
$$

Substituting (3.11) and (3.15) into (3.10), we see that

$$
\begin{aligned}
\left\|\left(P^{n}-h^{2 n} D\right) U\right\| & \geq\|U\| h^{2 n} \min \left\{-q-k^{2 n} \pi^{2 n}, \frac{(k+1)^{2 n} \pi^{2 n}+p}{2}\right\} \\
& =h^{2 n} d\|U\|,
\end{aligned}
$$

where $d=\min \left\{-q-k^{2 n} \pi^{2 n}, \frac{(k+1)^{2 n} \pi^{2 n}+p}{2}\right\}$.

Similarly, based on Lemma 3.2 and (3.16), it is easily checked that the matrix $P^{n}-h^{2 n} D$ is invertible and

$$
\left\|\left(P^{n}-h^{2 n} D\right)^{-1} U\right\| \leq \frac{1}{h^{2 n} d}\|U\| .
$$

In summary, we can state the convergence conclusion of the scheme (2.12) when $n$ is odd as follows.

According to the condition (1.2), when the step size $h$ satisfies (3.7) and (3.12), the matrix $P^{n}-h^{2 n} D$ is invertible, that is, (2.12) has a unique solution

$$
Z=\left(z_{j}\right)=\left(P^{n}-h^{2 n} D\right)^{-1} C_{n} .
$$

Then using (3.1), (3.9) and (3.17), we arrive at the estimate for the total truncation error $E=\left(e_{j}\right)$ of the scheme $(2.12)$

$$
\|E\|=\|Y-Z\|=\left\|\left(P^{n}-h^{2 n} D\right)^{-1} T_{n}\right\| \leq M h^{-2 n}\left\|T_{n}\right\|,
$$

where $M=\frac{2}{p+\pi^{2 n}}$ or $M=\frac{1}{d}$ is a constant depending only on $f(x)$. Also,

$$
\left\|T_{n}\right\|=\left(\sum_{j=1}^{N} t_{j}^{2}\right)^{\frac{1}{2}} \leq N^{\frac{1}{2}} \max _{1 \leq j \leq N}\left|t_{j}\right| \leq h^{-\frac{1}{2}} \max _{1 \leq i \leq N}\left|t_{i}\right|,
$$

so

(3.18) $\max _{1 \leq i \leq N}\left|e_{i}\right| \leq M h^{-2 n} h^{-\frac{1}{2}} \max _{1 \leq i \leq N}\left|t_{i}\right|=M h^{-2 n} h^{-\frac{1}{2}} O\left(h^{2 n+2}\right)=O\left(h^{\frac{3}{2}}\right)$.

It is seen that $\max _{1 \leq i \leq N}\left|e_{i}\right| \rightarrow 0$ as $h \rightarrow 0$, which implies that the numerical solution obtained from the scheme (2.12) converges to the exact solution of the $\operatorname{BVP}(1.1)$ and the total truncation error is $O\left(h^{\frac{3}{2}}\right)$.

Theorem. Suppose that $y(x)$ is the unique solution of the BVP (1.1) $(n \geq 2)$ under the condition (1.2) which is smooth sufficiently, the high-level derivative value does not change too rapidly, and the step size $h$ satisfies

$$
h \leq \min \left\{h_{1}, h_{2}\right\}
$$

where $h_{1}$ and $h_{2}$ satisfy (3.7) and (3.12), respectively. Then the scheme (2.12) has a unique solution $Z=\left(z_{i}\right)$ and its total truncation error is

$$
\max _{1 \leq i \leq N}\left|y\left(x_{i}\right)-z_{i}\right| \leq O\left(h^{\frac{3}{2}}\right) .
$$




\section{Numerical examples}

To illustrate the convergence and efficiency of the above scheme derived, we consider the following examples.

Example 1. Assume that a sixth-order two-point BVP is given in the form

$$
\left\{\begin{aligned}
y^{(6)}(x) & =f(x) y+g(x), 0<x<1, \\
y(0) & =1, \quad y^{\prime \prime}(0)=-1, y^{(4)}(0)=-3, \\
y(1) & =0, \quad y^{\prime \prime}(1)=-2 e, y^{(4)}(1)=-4 e,
\end{aligned}\right.
$$

where $f(x)=1, g(x)=-6 e^{x}$. The exact solution of this problem is $y=$ $(1-x) e^{x}$.

Since $p=\inf _{x \in[0,1]} f(x)=1>-\pi^{6}$, from (3.7) of case (1), the step size $h$ should satisfy

$$
h \leq h_{1}=\frac{2 \sqrt{6}}{\pi}\left[1-\left(\frac{1}{2}-\frac{1}{2 \pi^{6}}\right)^{\frac{1}{6}}\right]^{\frac{1}{2}}=0.51543984701666 .
$$

Table 1 gives the comparison of the numerical solution with the exact solution.

Table 1. Comparison of the numerical solution with the exact solution

\begin{tabular}{|c|c|c|c|}
\hline point $x_{i}$ & numerical solution $z_{i}$ & exact solution $y\left(x_{i}\right)$ & error $\left|y\left(x_{i}\right)-z_{i}\right|$ \\
\hline 0.0 & 1.00000000000000 & 1.00000000000000 & $0.00000000000000 \times 10^{-5}$ \\
\hline 0.1 & 0.99464075267847 & 0.99465382626808 & $1.307358961477600 \times 10^{-5}$ \\
\hline 0.2 & 0.97709732000550 & 0.97712220652814 & $2.488652263521463 \times 10^{-5}$ \\
\hline 0.3 & 0.94486686464972 & 0.94490116530320 & $3.430065348353217 \times 10^{-5}$ \\
\hline 0.4 & 0.89505441449065 & 0.89509481858476 & $4.040409410799306 \times 10^{-5}$ \\
\hline 0.5 & 0.82431804237927 & 0.82436063535006 & $4.259297079500790 \times 10^{-5}$ \\
\hline 0.6 & 0.72880689161249 & 0.72884752015620 & $4.062854371267299 \times 10^{-5}$ \\
\hline 0.7 & 0.60409114551398 & 0.60412581224114 & $3.466672716367913 \times 10^{-5}$ \\
\hline 0.8 & 0.44508292900531 & 0.44510818569849 & $2.525669318031998 \times 10^{-5}$ \\
\hline 0.9 & 0.24594700627127 & 0.24596031111569 & $1.330484442585433 \times 10^{-5}$ \\
\hline 1.0 & 0.00000000000000 & 0.00000000000000 & $0.000000000000000 \times 10^{-5}$ \\
\hline
\end{tabular}

From Table 1, it is clear that the error is considerately small. Inserting $\max _{1 \leq i \leq 9}\left|y\left(x_{i}\right)-z_{i}\right|=4.259297079500790 \times 10^{-5}$ into the error estimate (3.18), it should hold that

$$
\begin{aligned}
\max _{1 \leq i \leq 9}\left|y\left(x_{i}\right)-z_{i}\right| & \leq M h^{-6} h^{-\frac{1}{2}} \max _{1 \leq i \leq 9}\left|t_{i}\right| \\
& \leq \frac{2}{\pi^{6}+1} \times \frac{1}{4} \times 8 e \times 0.1^{\frac{3}{2}}=3.572759111412481 \times 10^{-4} .
\end{aligned}
$$

Obviously, the above inequality is correct, which means that the numerical results agree with the theoretical analysis.

The following Table 2 is a comparison of the numerical and theoretical errors for the method introduced in Section 2 under different steps. It shows that the numerical errors agree with the theoretical analysis. 
Table 2. Comparison of the numerical and theoretical errors

\begin{tabular}{|c|c|c|}
\hline step $h=\frac{1}{N+1}$ & error $\max _{1 \leq i \leq N}\left|e_{i}\right|$ & $O\left(h^{\frac{3}{2}}\right)$ \\
\hline$N=9$ & $4.259297079500790 \times 10^{-5}$ & $3.572759111412481 \times 10^{-4}$ \\
\hline$N=19$ & $1.055905343294583 \times 10^{-5}$ & $1.263161097612895 \times 10^{-4}$ \\
\hline$N=29$ & $4.685389965231046 \times 10^{-6}$ & $6.875778115745614 \times 10^{-5}$ \\
\hline$N=39$ & $2.626835689412133 \times 10^{-6}$ & $4.465948889265601 \times 10^{-5}$ \\
\hline$N=59$ & $1.186940417285953 \times 10^{-6}$ & $2.430954665788893 \times 10^{-5}$ \\
\hline$N=79$ & $8.633112595912706 \times 10^{-7}$ & $1.578951372016118 \times 10^{-5}$ \\
\hline
\end{tabular}

The following Table 3 exhibits the exact solution and the error estimates obtained by using the Adomian's decomposition method (ADM) [12], the homotopy perturbation method (HPM) [3], the variational iteration method (VIM) [4], and the finite difference method (FDM) introduced in this paper. It shows that the method obtained in this paper is very effective.

Table 3. Error estimates under different numerical methods

\begin{tabular}{|c|c|c|c|c|c|}
\hline$x_{i}$ & exact $y\left(x_{i}\right)$ & error to ADM & error to HPM & error to VIM & error to FDM \\
\hline 0.0 & 1.00000000 & 0.00000000 & 0.00000000 & 0.00000000 & $0.00000000 \times 10^{-5}$ \\
\hline 0.1 & 0.99465383 & 0.00040933 & 0.00040933 & 0.00040933 & $1.30735896 \times 10^{-5}$ \\
\hline 0.2 & 0.97712221 & 0.00077820 & 0.00077820 & 0.00077820 & $2.48865226 \times 10^{-5}$ \\
\hline 0.3 & 0.94490117 & 0.00107048 & 0.00107048 & 0.00107048 & $3.43006535 \times 10^{-5}$ \\
\hline 0.4 & 0.89509482 & 0.00125787 & 0.00125787 & 0.00125787 & $4.04040941 \times 10^{-5}$ \\
\hline 0.5 & 0.82436064 & 0.00132238 & 0.00132238 & 0.00132238 & $4.25929708 \times 10^{-5}$ \\
\hline 0.6 & 0.72884752 & 0.00125787 & 0.00125787 & 0.00125787 & $4.06285437 \times 10^{-5}$ \\
\hline 0.7 & 0.60412581 & 0.00107048 & 0.00107048 & 0.00107048 & $3.46667272 \times 10^{-5}$ \\
\hline 0.8 & 0.44510819 & 0.00077820 & 0.00077820 & 0.00077820 & $2.52566932 \times 10^{-5}$ \\
\hline 0.9 & 0.24596031 & 0.00040933 & 0.00040933 & 0.00040933 & $1.33048444 \times 10^{-5}$ \\
\hline 1.0 & 0.00000000 & 0.00000000 & 0.00000000 & 0.00000000 & $0.00000000 \times 10^{-5}$ \\
\hline
\end{tabular}

Example 2. Assume that the sixth-order two-point BVP is given in the form

$$
\left\{\begin{aligned}
y^{(6)}(x) & =f(x) y+g(x), 0<x<1, \\
y(0) & =2, y^{\prime \prime}(0)=0, y^{(4)}(0)=-2, \\
y(1) & =e, y^{\prime \prime}(1)=-e, y^{(4)}(1)=-3 e,
\end{aligned}\right.
$$

where $f(x)=-e^{-x}, g(x)=-(4+x) e^{x}+(2-x)$. The exact solution of this problem is $y=(2-x) e^{x}$.

Since $p=\inf _{x \in[0,1]} f(x)=1>-\pi^{6}$, from (3.7) of case (1), the step size $h$ should satisfy

$$
h \leq h_{1}=\frac{2 \sqrt{6}}{\pi}\left[1-\left(\frac{1}{2}-\frac{-1}{2 \pi^{6}}\right)^{\frac{1}{6}}\right]^{\frac{1}{2}}=0.5147106938533479 .
$$

Table 4 gives the comparison of the numerical solution with the exact solution. 
Table 4. Comparison of the numerical solution with the exact solution

\begin{tabular}{|c|c|c|c|}
\hline point $x_{i}$ & numerical solution $z_{i}$ & exact solution $y\left(x_{i}\right)$ & error $\left|y\left(x_{i}\right)-z_{i}\right|$ \\
\hline 0.0 & 2.000000000000000 & 2.000000000000000 & $0.000000000000000 \times 10^{-5}$ \\
\hline 0.1 & 2.099813385084812 & 2.099824744343731 & $1.135925891881939 \times 10^{-5}$ \\
\hline 0.2 & 2.198503341066027 & 2.198524964688306 & $2.162362227853265 \times 10^{-5}$ \\
\hline 0.3 & 2.294730168484334 & 2.294759972879205 & $2.980439487121345 \times 10^{-5}$ \\
\hline 0.4 & 2.386884407089619 & 2.386919516226033 & $3.510913641413538 \times 10^{-5}$ \\
\hline 0.5 & 2.473044893309774 & 2.473081906050192 & $3.701274041834068 \times 10^{-5}$ \\
\hline 0.6 & 2.550931013348714 & 2.550966320546712 & $3.530719799860194 \times 10^{-5}$ \\
\hline 0.7 & 2.617848392279256 & 2.617878519711620 & $3.012743236352478 \times 10^{-5}$ \\
\hline 0.8 & 2.670627163912752 & 2.670649114190961 & $2.195027820883766 \times 10^{-5}$ \\
\hline 0.9 & 2.705551858941608 & 2.705563422272645 & $1.156333103669027 \times 10^{-5}$ \\
\hline 1.0 & 2.718281828459046 & 2.718281828459046 & $0.000000000000000 \times 10^{-5}$ \\
\hline
\end{tabular}

Example 3. Assume that the sixth-order two-point BVP is given in the form

$$
\left\{\begin{aligned}
y^{(6)}(x) & =f(x) y+g(x), 0<x<1, \\
y(0) & =2, y^{\prime \prime}(0)=0, y^{(4)}(0)=-2, \\
y(1) & =e, y^{\prime \prime}(1)=-e, y^{(4)}(1)=-3 e,
\end{aligned}\right.
$$

where $f(x)=-3 \pi^{6} e^{-x}, g(x)=-(4+x) e^{x}+3 \pi^{6}(2-x)$. The exact solution of this problem is $y=(2-x) e^{x}$.

Since $p=\inf _{x \in[0,1]} f(x)=-3 \pi^{6}, q=\sup _{x \in[0,1]} f(x)=-3 \pi^{6} e^{-1}$, from (3.12) (here, $k=1$ ) of case (2), the step $h$ should satisfy

$$
h \leq h_{2}=\frac{2 \sqrt{6}}{2 \pi}\left[1-\left(\frac{1}{2}-\frac{-3 \pi^{6}}{2^{7} \pi^{6}}\right)^{\frac{1}{6}}\right]^{\frac{1}{2}}=0.2493485811548127 .
$$

Table 5 gives the comparison of the numerical solution with the exact solution.

Table 5. Comparison of the numerical solution with the exact solution

\begin{tabular}{|c|c|c|c|}
\hline point $x_{i}$ & numerical solution $z_{i}$ & exact solution $y\left(x_{i}\right)$ & error $\left|y\left(x_{i}\right)-z_{i}\right|$ \\
\hline 0.0 & 2.000000000000000 & 2.000000000000000 & $0.000000000000000 \times 10^{-5}$ \\
\hline 0.1 & 2.099837696498217 & 2.099824744343731 & $1.295215448671172 \times 10^{-5}$ \\
\hline 0.2 & 2.198549540308409 & 2.198524964688306 & $2.457562010338421 \times 10^{-5}$ \\
\hline 0.3 & 2.294793663811664 & 2.294759972879205 & $3.369093245852994 \times 10^{-5}$ \\
\hline 0.4 & 2.386958915636375 & 2.386919516226033 & $3.939941034181160 \times 10^{-5}$ \\
\hline 0.5 & 2.473123081690814 & 2.473081906050192 & $4.117564062200430 \times 10^{-5}$ \\
\hline 0.6 & 2.551005229817315 & 2.550966320546712 & $3.890927060279381 \times 10^{-5}$ \\
\hline 0.7 & 2.617911414246287 & 2.617878519711620 & $3.289453466726044 \times 10^{-5}$ \\
\hline 0.8 & 2.670672888774492 & 2.670649114190961 & $2.377458353031159 \times 10^{-5}$ \\
\hline 0.9 & 2.705575876608723 & 2.705563422272645 & $1.245433607754976 \times 10^{-5}$ \\
\hline 1.0 & 2.718281828459046 & 2.718281828459046 & $0.000000000000000 \times 10^{-5}$ \\
\hline
\end{tabular}




\section{References}

[1] A. Barari and M. Omidvar, An apprioximate solution for boundary value problems instructural engineering and mechanics, Math. Prom. Eng. 2008 (2008), no. 10, 1-10.

[2] M. K. Jain, S. R. K. Iyengar, and J. S. V. Saldanha, Numerical solution of a fourth-order ordinary differential equation, J. Engrg. Math. 11 (1977), no. 4, 373-380.

[3] M. A. Noor and S. T. Molyud-Din, A reliable approach for solving linear and nonlinear sixth-order boundary value problems, Int. J. Comput. Appl. Math. 2 (2007), no. 2, 163172 .

[4] M. A. Noor, K. I. Noor, and S. T. Mohyud-Din, Variational iteration method for solving sixth-order boundary value problems, Commun. Nonlinear Sci. Numer. Simul. 14 (2009), no. 6, 2571-2580.

[5] M. H. Pei, Existence and uniqueness of solutions to two-point boundary value problems for ordinary differential equations of order 2n, J. Systems Sci. Math. Sci. 17 (1997), no. 2, 165-172.

[6] R. A. Usmani, An $O\left(h^{6}\right)$ finite difference analogue for the solution of some differential equations occurring in plate deflection theory, J. Inst. Math. Appl. 20 (1977), no. 3, $331-333$.

[7] _ A uniqueness theorem for a boundary value problem, Proc. Amer. Math. Soc. 77 (1979), no. 3, 329-335.

[8] _ Discrete methods for boundary value problems with applications in plate deflection theory, Z. Angew. Math. Phys. 30 (1979), no. 1, 87-99.

[9] R. A. Usmani and M. J. Marsden, Numerical solution of some ordinary differential equations occurring in plate deflection thery, J. Engrg. Math. 9 (1975), no. 1, 1-10.

[10] _ Convergence of a numerical procedure for the solution of a fourth-order boundary value problem, Proc. Indian Acad. Sci. Sect. A Math. Sci. 88 (1979), no. 1, 21-30.

[11] X. Q. Wang, On a boundary value problem arising in elastic deflection theory, Bull. Austral. Math. Soc. 74 (2006), no. 3, 337-345.

[12] A. M. Wazwaz, The numerical solution of sixth-order boundary value problems by the modified decomposition method, Apple. Math. Comput. 118 (2001), no. 2-3, 311-325.

[13] C. Xu and S. Sun, Introduction to Computational Methods, Higher Education Press, Beijing, 2002.

[14] K. Zhang and Y. Zhao, Algorithm and Analysis of Numerical Computation, Science Press, Beijing, 2003.

Chenmei Xu

College of Mathematics and Information Science

HENAN UNIVERSITY

Kaifeng 475001, P. R. China

E-mail address: chen.m.x@henu.edu.cn

SHUAI JiAN

ACAdemy of Mathematics and Systems Science

Chinese ACAdemy of Sciences

Beijing 100080, P. R. China

BO WANG

College of Mathematics and Information Science

HenAN University

Kaifeng 475001, P. R. China 